\title{
Medical Perspective on Mental Health
}

\author{
Carol Harvey
}

\section{Contents}

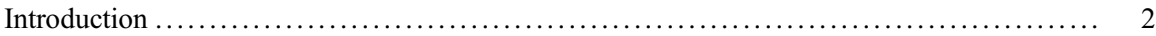

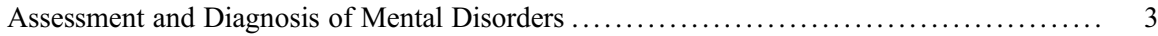

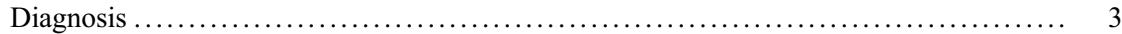

Providing a Diagnosis to Consumers and Families .............................. 8

Diagnosis as a Guide to Treatment and Service Access ......................... 10

Diagnosis as an Aid to Clinical Communication ................................ 11

The Narrow Medical or Brain-Disease Model of Mental Illness: History and Critiques ...... 11

The Biopsychosocial Model of Mental Illness .................................... 13

The Biopsychosocial Model in Practice ...................................... 14

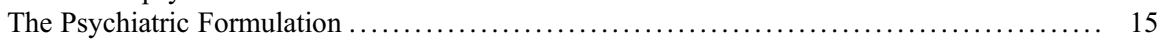

The Psychiatric Formulation in Mental Health Practice ............................ 16

The Place of Culture within Diagnosis, Formulation, and the Biopsychosocial Model ....... 17

Beyond the Brain-Disease Model: Other Aetiological Theories of Mental Illness .......... 17

Socio-developmental Influences on the Genesis and Course of Mental Disorder:

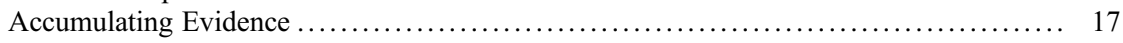

Current Understandings of Causation of Psychiatric Disorders: Gene-Environment

Interactions and Epigenetics ............................................... 19

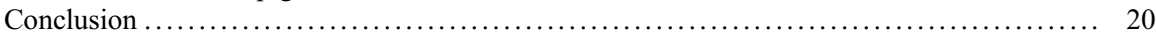

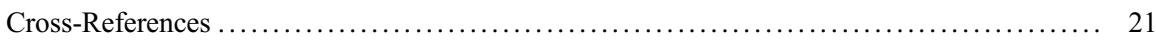

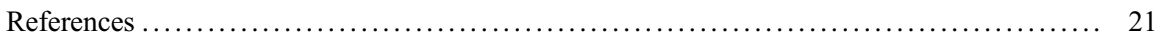

\begin{abstract}
This chapter provides a medical perspective on mental health, including current diagnostic systems which inform and underpin treatment and service delivery. Conceptualizations of mental disorders and their advantages and disadvantages for the assessment and treatment of persons living with mental illness are outlined. Despite its limitations, diagnosis remains central to communicating
\end{abstract}

\section{Harvey $(\triangle)$}

Department of Psychiatry, University of Melbourne and NorthWestern Mental Health, Melbourne, VIC, Australia e-mail: c.harvey@unimelb.edu.au 
a shared understanding of disordered experiences and behaviors between consumers, families, and professionals. Talking about a diagnosis in mental health is a skilled task, requiring sensitivity, an individualized approach within a trusting relationship, and sufficient time to address and revisit concerns and misunderstandings. All mental health professionals, including social workers, can helpfully contribute.

Current aetiological theories in mental health are described, including the medical or brain-disease model of mental illness which has been dominant in recent decades. Although not unique to psychiatry, the biopsychosocial (-cultural) model and psychiatric formulation both help to address some of the challenges of current diagnostic conceptualizations and to counterbalance the medical model. Further, these approaches are consistent with social work theory and practice and contribute strongly to a more person-centered, recovery-oriented approach to mental health practice which recognizes social aspects of human experience. Gene-environment interactions and epigenetics are also consistent with this since they integrate accumulating research evidence about social and environmental contributions to the development and course of mental disorders into aetiological theory. They also reaffirm the value of psychosocial interventions and person-in-environment approaches to recovery. The implications of these medical perspectives for social work practice in mental health are elaborated.

\section{Keywords}

Diagnosis · Biopsychosocial model $\cdot$ Formulation $\cdot$ Medical model $\cdot$ Braindisease model of mental illness $\cdot$ Social factors $\cdot$ Gene-environment interactions · Epigenetics

\section{Introduction}

This chapter will first focus on the psychiatric contribution to diagnostic systems in mental health. The underpinning conceptualizations of mental disorders and their associated advantages and disadvantages and the challenges and solutions these present for the assessment and treatment of persons living with mental illness will be outlined. The importance of the biopsychosocial (-cultural) model, and of formulation, will be elaborated as a way of addressing some of the challenges represented within current diagnostic conceptualizations. An overview of current aetiological theories in mental health will also be provided, including consideration of the medical or brain-disease model of mental illness. Other theories which link with broader conceptualizations of causation and emphasize findings derived from social psychiatric research and recent work in gene-environment interactions and epigenetics will be briefly described. The implications of these medical perspectives on mental health for social work practice will be elaborated. 
The term "consumer" is used throughout this chapter to refer to people living with mental illness of various types. This is consistent with the widespread use of this term in Australian mental health services. However, it is acknowledged that other terms such as "patient," "client," or "service user" are in more common usage elsewhere. The term "patient" will also be used if also used in the source material.

\section{Assessment and Diagnosis of Mental Disorders}

A key contribution of medical practitioners to multidisciplinary mental health practice is their expertise in assessment and diagnosis of mental disorders. Consequently, there is a strong emphasis on knowledge and skills relevant to this area of practice in psychiatric training (Chaplin et al. 2007).

\section{Diagnosis}

There are many reasons for accurate diagnosis in mental health, although most agree that the major reason is to guide treatment planning (Chaplin et al. 2007; Outram et al. 2014). There are two major diagnostic classification systems in widespread use in mental health internationally: the Diagnostic and Statistical Manual of Mental Disorders (DSM) developed by the American Psychiatric Association (APA) and the International Classification of Diseases (ICD) which is published by the World Health Organization (WHO).

\section{Brief Overview of the Two Major Diagnostic Systems}

The classification of mental disorders was included in the 8th edition of the ICD in the 1970s (Singh et al. 2012). The ICD is in its 10th edition (World Health Organisation 1992), an edition first endorsed in 1990 and regularly updated but soon to be replaced by version 11 . According to the WHO ICD website, the ICD is "used in clinical care and research to define diseases and study disease patterns, as well as manage health care, monitor outcomes and allocate resources." The ICD provides a common language for the identification of health trends and statistics globally (http://www.who.int/classifications/icd/factsheet/en/).

The DSM has existed in various versions since 1952; DSM-5 was released in 2013 (American Psychiatric Association 2013). The APA DSM website describes the DSM as "an authoritative volume that defines and classifies mental disorders in order to improve diagnoses, treatment, and research" (https://www.psychiatry.org/ psychiatrists/practice/dsm).

Both these major diagnostic classification systems have drawn on expert scientific advice. The development of earlier versions of the mental disorder classification within ICD was linked to large multicountry epidemiological studies such as the International Pilot Study of Schizophrenia (Leff et al. 1992). In the case of each 
DSM revision, expertise is drawn from numerous research scientists from psychiatry and other disciplines, clinical care providers, and consumer and family advocates. For DSM-5, relevant research was evaluated by a task force and work groups to propose draft criteria which were finally approved after expert advice on the strength of evidence for proposed changes and their clinical utility and public health impact.

\section{Categorical and Dimensional Approaches to Diagnosis}

A weakness of current diagnoses in mental health is the lack of corresponding evidence about the physiological processes or mechanisms, whereby such conditions develop and progress. So, there is a lack of laboratory tests or other investigations to inform most diagnoses (Nemeroff et al. 2013; Nesse and Stein 2012; Timimi 2014). Therefore, diagnostic classification systems in mental health are symptom-based and rely heavily on the recognition of syndromes - a collection of symptoms (and signs) that occur together as an identifiable condition in a group of people.

Psychiatric syndromes are complex phenomena which can potentially be conceptualized and assessed in two contrasting ways: either as categories or as dimensions. Categorical approaches to diagnosis tend to diagnose a case of a disorder at a threshold number of symptoms. For instance, major depressive disorder is diagnosed in DSM-5 when a person reports that five (or more) of nine possible symptoms have been present during the same 2-week period (American Psychiatric Association 2013). The most obvious advantage of this approach is that it assists clinicians to decide who is sufficiently ill to require treatment, by applying this categorical "cut point." However, observations from both clinical practice and research reveal that there is no separation at this "cut point" between "non-cases" and "cases" of disorder, as expected within a categorical model of diagnosis; in other words, a number of people score just below the threshold for disorder ("subthreshold cases"). Therefore, "zones of rarity" between mental disorders which indicate clear separations between each syndrome are rarely observed, and it is difficult to draw clear boundaries between many disorders and normality (Nesse and Stein 2012; O'Donovan and Owen 2016). These observations fit better with a dimensional approach to diagnosis (described more fully below).

Categorical diagnoses are arguably most valuable for homogeneous disorders, for example, diagnoses where symptom profiles and severity are similar, where aetiological factors are shared, and where categorical diagnosis guides treatment with some accuracy (Macneil et al. 2012). However, few psychiatric disorders match this description (Nesse and Stein 2012; O'Donovan and Owen 2016). As these and other authors have argued, two individuals with major depression may have quite different symptom profiles and severity. Conversely, symptoms such as auditory hallucinations can be shared across several diagnoses, for example, schizophrenia and mania with psychotic features. Further, phenomena as diverse as mood and personality disorders, psychoses, and anxiety disorders can be associated with varied aetiological factors, ranging from genetic to environmental. These may include trauma, personality styles, and interpersonal stressors, with each factor playing a greater or lesser role for each person (Macneil et al. 2012). Thus, a categorical approach is not only a less than perfect fit for the observed phenomena but also has the added disadvantage that it may lead clinicians to overlook the complexity and 
heterogeneity inherent in an individual's symptomatology or within the aetiological factors for their disorder.

Another important limitation of the categorical approach to diagnosis is that many individuals are found to have more than one disorder, that is, to experience comorbidity. Thus, it appears necessary in clinical practice to make more than one diagnosis to encompass the experiences and difficulties of a significant minority of people. Epidemiological surveys provide support for these clinical observations. For example, analyses of the 2007 Australian National Survey of Mental Health and Well-Being (Teesson et al. 2009) show that $25 \%$ of all cases of the common mental disorders were comorbid with at least one other disorder.

Given the difficulties raised by a categorical approach to diagnosis, there is increasing interest in applying a dimensional approach to conceptualizing and assessing psychiatric syndromes. A dimensional approach classifies mental disorders by quantifying a person's symptoms or other characteristics of interest and representing them with numerical values on one or more scales or continuums, rather than assigning them to a mental disorder category (Nesse and Stein 2012). Thus, traits such as mood or interpersonal functioning exist on a continuum ranging from normal variation to pathology (Tyrer et al. 2011). As mentioned, this better describes the existence of subthreshold cases of various disorders. Subthreshold cases are important to encompass within any diagnostic approach since they involve impaired functioning and may result in appropriate clinical referral and need for services (e.g., Rutter 2011). However, there are also potential problems with a dimensional approach, including the generation of multiple combinations of high and low scores on each of a number of dimensional characteristics, which makes the system unwieldy and impractical (Tyrer et al. 2011).

Both major diagnostic systems use categorical and dimensional approaches, although relying more heavily on categorical. These two approaches should be seen as complementary. Thus, dimensional assessments allow for more fine-grained descriptions which may often inform the more user-friendly categorical approach to treatment decisions.

\section{Overall Critique of Current Diagnostic Systems in Mental Health}

Many critiques emphasize the conceptual and empirical limitations of diagnostic systems in mental health, although these have particularly focussed on the latest version of the DSM (e.g., Frances 2012; Pemberton and Wainwright 2014; Timimi et al. 2014). These have come from within psychiatry as well as other disciplines, including social work (e.g., see the special issue of Research on Social Work Practice, including Lacasse 2014a). It is important that social workers are informed about current diagnostic systems and their advantages and disadvantages (see Table 1).

A recurring criticism of the main systems of psychiatric diagnosis is that disorders are reified, that is, something subjective is turned into something "concrete," despite the noted limitations of classificatory systems. Also, links between life experiences and symptoms of emotional distress can be lost or obscured (Humphreys and Thiara 
Table 1 Implications for social work practice

It is essential for social workers to be well informed about current diagnostic systems and their conceptual underpinnings and the implications of these for the individual consumer, their family, and the service system, as well as for policy, funding, and research

Social workers should understand the main advantages and disadvantages of diagnostic systems and diagnoses, so as to adopt an appropriately critical stance to their application within practice

Understanding the wide range of responses of consumers and families to receipt of a diagnosis and how and why these occur is essential for social work practice

Social workers can play a helpful supporting role in the provision of a diagnosis to a consumer and their family, grounded in their acquisition of effective communication skills and the application of these in team-based approaches

The biopsychosocial model and psychiatric formulation represent useful knowledge and skills sets for all mental health professionals, including social workers

Social workers should ensure that the "bio-" continues to be part of their application of the biopsychosocial model and of person-in-environment approaches within their practice

Social workers can play an important role by advocating for, and applying their understanding of, the wider context of mental disorder within the practice of diagnosis and treatment; this includes contributing person-in-environment perspectives

Advances in the understanding of biomedical contributions to the aetiology of mental disorders, especially gene-environment interactions and epigenetics, should prompt social workers to rethink their knowledge base

Social workers should aim to be informed, but not expert, readers of biomedical developments

The integration of biological and social perspectives, underpinned by robust research, is essential, and social workers have an important contribution to make to this

Advocacy for ethical and person-centered translation of emerging scientific knowledge and the protection of vulnerable populations continues to be a very important role for social workers

2003; Lacasse 2014b; Pemberton and Wainwright 2014; Timimi 2014; Wong 2014). Further, clinicians may be distracted from other relevant symptoms and contributory factors, including their complexities and interactions, which are not described within the operationalized diagnostic criteria for each disorder (Macneil et al. 2012; Wong 2014). For instance, Humphreys and Thiara (2003) argue that an exclusively medical model approach to diagnosis for women with mental health symptoms due to domestic violence may result in pathologizing the individual rather than acknowledging and addressing the abuse context. This decontextualized view of behavior, in which problems in living are medicalized, may have dehumanizing and unhelpful consequences in terms of treatment and service responses (Frances 2012; Humphreys and Thiara 2003; Lacasse 2014b). One way of attempting to better represent the complex nature of psychiatric disorders was the multiaxial diagnostic assessment system introduced in DSM-3. Thus, each individual could be evaluated in terms of several different domains of experience which went beyond acute symptoms to try to encompass the diverse factors that account for a patient's mental health. This multiaxial system included Axis IV - sometimes referred to as the social work axis - which could highlight psychosocial and environmental problems. Unfortunately, the multiaxial system was removed from DSM-5 (Lacasse 2014b). 
The validity of psychiatric diagnoses, that is, whether they represent actual entities, is undermined by the existence of widespread comorbidity, as previously described. Many authors have pointed out that the reliability of many psychiatric diagnoses is poor and this is especially the case in clinical settings (Lacasse 2014b; Timimi 2014). Thus, two clinicians seeing the same patient independently will not reach the same diagnosis on most occasions. Inter-rater agreements were reported to be uniformly poor for some common diagnoses such as major depressive disorder and generalized anxiety disorder in DSM-5 field trials, a finding linked with the marked heterogeneity of people who meet criteria for these disorders and comorbidity between these and other disorders (Lacasse 2014b; Regier et al. 2013; Timimi 2014).

The sheer number of diagnoses in DSM has been criticized: DSM-1 listed 106 diagnoses and DSM-5 describes 157. In part, this represents efforts to increase the coverage of the system by identifying new disorders or specific subtypes of existing disorders with purported treatment or other clinical implications. The aim is to explain more of the observed phenomena and, through refinement of diagnoses, to lead to more appropriate and effective treatments. However, one adverse consequence is the creation of circumstances in which comorbid disorders are increasingly common. Most would agree that the number of diagnoses is far too many for any clinician to remember the criteria for each, which arguably undermines the usefulness of the system (Nemeroff et al. 2013). Moreover, some critics have linked the number of diagnoses in DSM with the financial (as opposed to scientific) utility of DSM-5 (Frances 2012; Lacasse 2014b). Concern has also been raised that this represents a medicalization of human problems and/or problems of daily living (Frances 2012; Lacasse 2014b). A frequently cited example is the removal of bereavement as an exclusion criterion for the diagnosis of major depression. This challenges the view that grief after the loss of a loved one, which frequently comprises depressive symptoms, belongs to the category of healthy psychic reactions and coping strategies (Frances 2012; Nemeroff et al. 2013).

Notwithstanding these challenges and difficulties in assigning reliable and valid diagnoses in mental health, formulating diagnoses continues to be a very important activity for pragmatic reasons. Diagnosis assists with collecting data at a service or population level - a purpose for which the ICD classification is explicitly designed (http://www.who.int/classifications/icd/factsheet/en/). This can be useful for service planning, benchmarking between services, and research, especially epidemiological research on variations in prevalence and outcome of mental disorders and their associated biological, psychological, and social factors. Further, receipt of a diagnosis by an individual may facilitate access to treatment, services, and resources for the individual as well as their family, as well as enabling funding for specific treatments and medication subsidies (Frances 2012; Nemeroff et al. 2013). Making and providing a diagnosis to consumers and their families is therefore a significant contribution of medical practitioners in mental health. However, providing a meaningful diagnosis in a timely and sensitive manner is by no means a uniformly shared and applied knowledge and expertise. 


\section{Providing a Diagnosis to Consumers and Families}

In their review article, Milton and Mullan (2014) describe how rates of provision of diagnoses to consumers have increased in the last decade and how they vary by diagnosis and probably by culture and migrant status. Thus, diagnostic discussion for schizophrenia diagnoses and for general mental health diagnoses varies between $77 \%$ and $88 \%$, except for consumers who had immigrated (22\%). Communicating a schizophrenia diagnosis appears less common than, say, providing a depression diagnosis, and a substitute or euphemistic diagnosis such as psychosis or major mental illness may be provided for the former (Outram et al. 2014). This is associated with consumers having a poorer understanding of their condition (Milton and Mullan 2014). While most clinicians in an Australian study supported the need to give a diagnosis of schizophrenia, they gave multiple reasons for not doing so in practice (Outram et al. 2014); psychiatrists interviewed in a separate study even thought it might be anti-therapeutic to discuss diagnosis when prescribing antipsychotics (Chaplin et al. 2007). These perplexing and contradictory findings raise important questions about what should be a fundamental practice in mental health care.

In part, clinicians' varied responses and practices concerning provision of a diagnosis may relate to their awareness of the diverse reactions of consumers and their families to receiving a diagnosis. It cannot be assumed that receipt of a diagnosis is either "good or bad news" (Gallagher et al. 2010), since reactions are complex and vary between individuals provided the same diagnosis and between individuals according to diagnosis; for example, there are more negative responses to a diagnosis of Alzheimer's disease compared with vascular dementia (Aminzadeh et al. 2007).

Recognized negative reactions include anger, anxiety, fear, despair, shock, denial, and shame, the latter especially due to cultural beliefs. Some of these reactions relate to concerns about feelings and experiences of stigma, with more widespread concerns among those receiving diagnoses of psychotic, as opposed to nonpsychotic, disorders (Dinos et al. 2004; Mestdagh and Hansen 2014; Milton and Mullan 2014). The negative impact of a mental illness diagnosis on an individual's social identity and relationships with others is regularly reported and may undermine their willingness to share diagnostic information with others and/or lead to selective hiding of their diagnosis or even avoidance of contact with others (Mestdagh and Hansen 2014; Milton and Mullan 2014). Nevertheless, the importance of accuracy in diagnosis is emphasized. For example, parents of children with autism have reported that confusion may be experienced in the context of misdiagnosis, which is common in the early stages of assessment (Altiere and Von Kluge 2009). However, even when technically correct information is given, such as more than one diagnosis when this is consistent with comorbidity in current diagnostic systems, consumers have reported confusion (Gallagher et al. 2010). A diagnosis may be rejected because it pathologized issues or did not help individuals make sense of their situation (Humphreys and Thiara 2003; Milton and Mullan 2014). This may link with 
previously described deficiencies of existing diagnostic systems. Finally, and despite the importance of diagnoses in enabling treatment access, treatment dropouts have been reported because of hearing a diagnosis (Milton and Mullan 2014).

On the other hand, there is potential relief for the consumer in having a diagnosis to help explain what they have been experiencing. This is because the initial onset of illness may involve confusing and/or frightening changes such as distressing symptoms, isolation from friends and family, difficulties in everyday functioning, or a long-standing sense of difference from others. This sense of relief may apply equally to family members. Relief in response to a diagnosis is most apparent in people diagnosed with depression or anxiety (Milton and Mullan 2014; Dinos et al. 2004). Nevertheless, most research with people with schizophrenia (and their families) suggests a preference to have a named diagnostic entity rather than uncertainty (Magliano et al. 2008). Similarly, some adolescents interviewed by Buston (2002) spoke about a lack of diagnosis being frustrating and leading to uncertainty, even though clinicians reported greater reluctance to give younger people a diagnosis because of stigma-related concerns (Outram et al. 2014). Other positive responses include viewing the diagnosis as validating or helpful to treatment and presenting an opportunity to develop positive coping skills (Aminzadeh et al. 2007; Milton and Mullan 2014). Overall, the review of Milton and Mullan (2014) concluded that most consumers want diagnostic information and see it as their right choice. Therefore, current practice suggests a gap between the information preferences of consumers and their level of satisfaction with diagnosis discussion (Fossey et al. 2012; Milton and Mullan 2014).

There is increasing recognition that providing a diagnosis requires an individualized approach to giving information in the context of a trusting relationship and with active involvement of the consumer and their family and key supports wherever possible (Gallagher et al. 2010; Mestdagh and Hansen 2014). This should include discussion about the meaning and implications of the diagnosis, so as to avoid misunderstanding and help the consumer and their family to develop a realistic appraisal of their situation. It is apparent that this requires time (Gallagher et al. 2010) and is often an ongoing process of care rather than a one-off event, as exemplified in the disclosure of a dementia diagnosis (Aminzadeh et al. 2007). It requires mastery of the "subtle interplay of hope and pessimism" in communication of diagnosis (Outram et al. 2014, page 551). While focussed on reported practices of consultant psychiatrists when prescribing antipsychotics, Chaplin et al. (2007) provide useful strategies for strengthening the therapeutic alliance in the context of potentially difficult discussions with consumers. Intervention studies aimed at facilitating diagnostic communication are still few but showed significant improvements in consumer satisfaction and mood and in clinician communication skills, without negative outcomes for consumers or clinicians (Holm-Denoma et al. 2008; Milton and Mullan 2014). Further, satisfaction with diagnosis is higher if it is received face-to-face and if there is supplementary support from other professionals (Milton and Mullan 2014), suggesting a specific and important role for social workers (see Table 1). There are growing recommendations for development of detailed protocols for communicating diagnosis, including coordinated team-based 
approaches, and to feed these into training (Chaplin et al. 2007; Milton and Mullan 2014). However, more research is needed to achieve this aim, including with more professional groups and with greater inclusion of participants with psychosis and taking cross-cultural issues into consideration (Aminzadeh et al. 2007; HolmDenoma et al. 2008; Milton and Mullan 2014).

\section{Diagnosis as a Guide to Treatment and Service Access}

As already emphasized, diagnosis can be useful to guide treatment and is commonly perceived as the main purpose of diagnosis. Current psychiatric medications are mostly symptomatic treatments, as is consistent with the largely symptom-based nature of psychiatric diagnoses. As argued by Nemeroff et al. (2013), helpful associations between treatment efficacy and existing diagnostic boundaries do exist: for example, lithium is very effective for many people with a diagnosis of bipolar disorder but is not an effective antipsychotic. However, it should also be noted that other treatments are helpful for more than one diagnosis, for example, antipsychotics may be effective treatments for schizophrenia and mania (Nemeroff et al. 2013).

Provision of a diagnosis may facilitate access to diagnosis-specific services that are available for people with autism or dementia. Individuals without a definitive diagnosis may therefore be excluded from needed services and resources, such as access to supports, benefits, or medication subsidies (Milton and Mullan 2014; Milton and Mullan 2017). Some mental health services are organized according to diagnosis-specific care pathways (e.g., borderline personality disorder) to improve quality of care and efficiency through the systematic application of treatment recommendations in clinical practice guidelines (e.g., National Institute for Health and Care Excellence 2009). Whether diagnostic (and therefore eligibility) criteria are met therefore becomes an important issue. For example, it has been argued that changes within DSM-5 to the diagnosis of autism will affect access to services such that those with "higher-functioning autism" may lose out (see Nemeroff et al. 2013).

Particularly important is the role of diagnosis in facilitating access to early intervention. Most would agree that early intervention is a worthwhile goal; nevertheless, the practicalities are not straightforward (van Os and Guloksuz 2017; Yung and Nelson 2013). By way of example, it has been argued that the existence of a prodromal phase in most cases of schizophrenia (i.e., changes or deterioration in diverse subjective symptoms and behavioral signs that precede the onset of psychotic symptoms) creates a target for early intervention. The rationale is that early intervention would prevent, ameliorate, or delay onset of psychosis (Yung and Nelson 2013). Criteria for "ultrahigh risk" (UHR) or "clinical high risk" (CHR) for "transition" to a psychotic disorder have been developed, with accompanying treatments and services. Treatments remain controversial, however, due to concerns that a substantial proportion of people not truly at risk (false positives) may be treated, including with antipsychotic medications which carry attendant risks of side effects (Frances 2011; Yung and Nelson 2013). The latest evidence does not support 
use of antipsychotics in the UHR group (Stafford et al. 2013; Yung and Nelson 2013); even so, the concept of an ultrahigh-risk diagnosis continues to be debated and has not been formally included in DSM-5 (Frances 2011; Frances 2012; Nemeroff et al. 2013; van Os and Guloksuz 2017). Van Os and Murray (2013) question whether the ultrahigh-risk state is a reliable and valid binary concept. They also question whether treatment of this condition can prevent the transition to frank psychosis, which may be an invalid or overly simplistic concept (van Os and Murray 2013). Instead, they and others argue for a public health perspective rather than the relatively inefficient medical high-risk approach (van Os and Guloksuz 2017). Thus, while the importance of timely access to appropriate services and resources is largely undisputed, whether diagnosis is accurate and meaningful in so far as providing a helpful therapeutic target is clearly a crucial prerequisite.

\section{Diagnosis as an Aid to Clinical Communication}

Diagnosis may facilitate communication between clinicians and provide a useful shorthand for conveying information and orientating all concerned to relevant considerations, such as treatment options and prognosis (e.g., American Psychiatric Association 2013). On the other hand, Lacasse (2014a) has argued that unreliable diagnostic labels are probably unhelpful in clinical communication. Further, shorthand descriptions such as diagnosis carry the risk of overlooking the rich and nuanced details of each person's situation and may lead to professionals treating all persons with a specific diagnosis as being the same, with identical issues and needs. Despite these caveats, diagnoses continue to be "invaluable working concepts for clinicians" (Kendell and Jablensky 2003). Also, person-in-environment perspectives contributed by social workers can assist with mitigating this risk (see Table 1). Further, there are several important conceptual developments which, when applied in practice, mitigate the potentially adverse consequences of applying diagnoses in mental health practice and represent an important medical contribution. These are elaborated below - see sections "The Biopsychosocial Model of Mental Illness" and "The Psychiatric Formulation."

\section{The Narrow Medical or Brain-Disease Model of Mental Illness: History and Critiques}

The brain-disease model of mental illness has been dominant in recent decades and underpins current research and approaches to diagnosis. Neurobiological models of mental illness are too varied to be satisfactorily summarized here. However, a brief overview of the most common and enduring conceptualization, the dopamine hypothesis of schizophrenia, will be provided. Kendler and Schaffner (2011) have written a useful account of the history of the dopamine hypothesis of schizophrenia, pointing out that this has been one of the most prominent aetiological theories in psychiatry for over 50 years. Dopamine is one of a number of neurotransmitters, that 
is, chemical mediators between nerve cells in the brain. In its earliest form, the dopamine theory proposed that schizophrenia may be related to a relative excess of dopamine-dependent neuronal activity in the brain (Meltzer and Stahl 1976). A selective synthesis of meta-analyses and reviews of studies attempting to validate the dopamine hypothesis, ranging from measuring brain levels of dopamine and its metabolites to genetic association studies, is summarized by Kendler and Schaffner (2011). They conclude that efforts to empirically validate the hypothesis have largely failed, leading to it being reformulated.

The current and most accepted reformulation of the dopamine hypothesis is that schizophrenia is characterized by reduced dopamine in the frontal areas of the brain and excessive dopamine in neurons in the mesolimbic pathway, sometimes referred to as the reward pathway (Davis et al. 1991). The former dysregulation is postulated to lead to negative symptoms of schizophrenia, such as cognitive impairments, and the latter to positive symptoms, such as hallucinations. Further, experts increasingly agree that dopamine does not explain the cause of schizophrenia per se, rather it acts as the final common pathway of diverse predisposing factors, environmental or genetic or both (Howes et al. 2017; Kendler and Schaffner 2011; Lau et al. 2013). This is consistent with the pharmacological dopamine hypothesis of antipsychotic action for which there is more substantial support. Kendler and Schaffner (2011, p. 59) point out that evidence for "other leading 'neurochemical' aetiological theories in psychiatry, such as the serotonin hypothesis of depression," may be similarly lacking.

There have undoubtedly been many advances in understanding the neurobiology of psychiatric disorders, for instance, through brain imaging studies. However, the narrow conception and/or application of a medical, and especially brain disease, model of mental illness has received many criticisms (e.g., Healy 2016; Malla et al. 2015; Timimi 2014; Wakefield 2013; Wong 2014). It has been argued that a braindisease model of mental illness would reduce stigma and accusations that people with mental illness are weak-willed or have a character defect but it appears that the contrary is true (Timimi 2014; Wakefield 2013). It seems that the public do not have an explanatory model of mental illness as "just like medical illness," and, contrary to previous assumptions, those who adhere to such a model are less optimistic and accepting of people with mental illness (Malla et al. 2015).

Malla et al. (2015) have also argued that a neurobiological model of mental illness is unhelpful in other respects. Unlike somatic illnesses such as diabetes, mental disorders "affect the very core of one's being through a range of experiences and phenomena of varying severity that alter the individual's thinking, perception and consciousness about the self, others and the world" (Malla et al. 2015, p. 148). Thus, therapy goes beyond restoring dysfunctional physiological mechanisms and encompasses helping the person feel better and to interact more adaptively with their physical and social environments (Malla et al. 2015). A brain-disease model is arguably not helpful for acceptance of psychological and social treatments (Malla et al. 2015; Wong 2014) and, moreover, may lead to overuse of medication (Wakefield 2013). Indeed, it has been argued that overreliance on a medical model is associated with poor treatment outcomes in the current service system (Pemberton and Wainwright 2014; Timimi 2014; Wong 2014). 
The example of evolving models for understanding alcohol addiction or dependence provides a useful illustration of the dilemmas posed by over-adherence to a brain-disease model of mental illness. In the nineteenth century, a moral model of addiction was evident which held people responsible for their behavior and tended to be applied in a harshly moralistic or punitive manner (see Elam 2015; Morse 2004). It was argued that application of a chronic brain disease or medical model to alcohol (and other) addictions would alleviate stigma, reduce "victim-blaming," and encourage many to seek help (Ferentzy and Turner 2012) since the condition was then seen as wholly outside their control. However, one of the adverse consequences of this model is to remove any idea of the drinker as an active participant in their recovery (Peele 2016). Recent research contradicts this in so far as it reveals that change in addictive behavior is usually self-initiated (Peele 2016). Further, motivational interviewing approaches have been shown to enhance consumers' intention to change and are now regarded as evidence-based practices in the substance abuse field (Lundahl et al. 2010). A relevant conclusion in this field, but also more broadly applicable in mental health, is that while the medical, and more particularly the brain disease, model should not be abandoned, its narrow application is unhelpful (Healy 2016).

\section{The Biopsychosocial Model of Mental Illness}

Despite the recent dominance of the brain-disease model of mental illness, other models of mental disorder have also existed for some time and may be considered to complement (rather than replace) the brain-disease model. They have helped to reposition the person experiencing the mental disorder as a more active participant in their own recovery, particularly by emphasizing the centrality of the person's experiences in understanding psychiatric disorders (Healy 2016; Pemberton and Wainwright 2014). A good example is the biopsychosocial model which has been influential within psychiatry for some time. Detailed histories and descriptions of the biopsychosocial model are provided in most psychiatry and mental health textbooks (e.g., Bland et al. 2012, pp. 399-400). The term owes a great deal to the American psychiatrist George Engel. He suggested that the biological, psychological, and social dimensions of illness must be attended to simultaneously rather than taking a narrow medical approach to mental illness (Borrell-Carrió et al. 2004; Engel 1977). Further, Engel's model implied that biological, psychological, and social elements and factors interact dynamically, in health and in illness, and emphasized a systems view of health and illness, with each system forming part of higher-order systems (Engel 1977; Garland and Howard 2009; Healy 2016). Thus, systems extended from cells, tissues, and organs through an individual person and beyond to a family, a community, culture and subcultures, and society (Bland et al. 2012; Borrell-Carrió et al. 2004; Farre and Rapley 2017). Reciprocal interactions between biological, psychological, and social factors and the multiple levels of the various systems are conceptualized, consistent with models of circular rather than linear causality. 
This conceptualization has underpinned other models, such as the World Health Organization's (2001) International Classification of Functioning, Disability and Health (http://www.who.int/classifications/icf/en/ accessed 19 April 2018).

There are many potential implications of applying the biopsychosocial model in practice. It is widely accepted that the biopsychosocial model can guide clinicians in their everyday work and has the potential to lead to more humanistic and patientcentered care (Borrell-Carrió et al. 2004; Farre and Rapley 2017). The model has helped to include consideration of emotional wellbeing, functioning in daily life and performance of social roles within patient assessment and care, which are particularly important in chronic disease (Fava and Sonino 2008; World Health Organisation 2001). The model is more consistent with the recovery movement and consumer demands for more psychologically based mental health care, as well as with a public health orientation to mental health (Pemberton and Wainwright 2014). Consistent with social work practice (Healy 2016), the biopsychosocial model underlines the importance of understanding the person in their context (Timimi 2014) which is central to the person-in-environment approach (Wong 2014). Implicit in these approaches is the involvement of the patient's relatives and/or significant others in assessment and treatment planning because of their often-extensive knowledge of the patient as well as their significance for the person's recovery (Harvey and O'Hanlon 2013).

The biopsychosocial model (and related approaches which emphasize the relevance of psychological and social factors, as well as biological factors, to the causation and outcome of mental illness) is consistent with accumulating evidence for socio-developmental influences on the genesis and course of mental disorder. Evidence is comprehensively outlined in other texts (e.g., see "Principles of Social Psychiatry," Morgan and Bhugra 2010) and will be briefly summarized later - see section "Beyond the Brain-Disease Model: Other Aetiological Theories of Mental Illness."

\section{The Biopsychosocial Model in Practice}

Concerns about the biopsychosocial model have been raised. These include that it is imperfectly applied in day-to-day practice and the end result is often to preference one aspect of the model over another. So, biological considerations may still dominate in some settings, or psychoanalytic concepts may be preeminent elsewhere (Ghaemi 2009). Within social work (see Table 1), some have argued that the "bio-" has been ignored (e.g., Maynard et al. 2017). Some critics identify deficiencies in the model itself and argue these explain its imperfect implementation. These include that the model is too vaguely defined and therefore not testable; does not include a method to identify relevant biopsychosocial data; gives no indication of what level of analysis (biological, psychological, or social) to prioritize and when; and cannot be efficiently put into practice because it is too time-consuming (Farre and Rapley 2017; Ghaemi 2009). Ghaemi (2009) has argued that the model is of limited value since it is so vague as to lead to dogmatic pursuit of eclectic and nonevidence-based 
approaches. Modifications or enhancements of the model have been proposed which include emphasizing cultural considerations (which were always a component of Engel's model) or proposing patient-centered interviewing methods which could be used to elicit relevant biopsychosocial data (Engel 1977; Farre and Rapley 2017; Smith et al. 2013). Despite the identified drawbacks, however, the biopsychosocial model continues to influence much psychiatric practice and is routinely used as a guiding framework for formulation, another significant component of psychiatric assessment and treatment planning.

\section{The Psychiatric Formulation}

The information gleaned from the clinical activities of history-taking and mental state and physical examination, which underpin the generation of a diagnosis as a fundamental part of psychiatric practice, may be summarized and then synthesized to construct a formulation. Diagnosis and formulation are essential components of psychiatric practice, and formulation enhances the value of diagnosis and may serve to mitigate some of its shortcomings. As previously argued, a diagnosis in isolation tells us little about a person's experience of mental disorder, fails to take account of current and historical environmental events, and typically overlooks external causal or contributory factors (Macneil et al. 2012; Wong 2014). Therefore, it is relevant to identify and understand these broader aspects of experience to guide a more individualized and personalized approach to treatment planning and select potential targets for therapeutic change (de Beer 2017; Macneil et al. 2012; Wong 2014). One way to achieve this is to generate a formulation.

A formulation goes beyond a summary and attempts a synthesis of available clinical information by concentrating on those aspects of the history that appear most significant and including some understanding of the interactions between events (de Beer 2017; Kirby and Grover 2017; Meadows et al. 2012). It aims to identify and explain aetiological factors (de Beer 2017). Further, formulation synthesizes the patient's experience with relevant clinical theory and research and thereby forms the bridge between assessment and treatment (Macneil et al. 2012). Thus, the selection of information and the inferences drawn from it depend on the theoretical framework used and the evidence which informs the formulation (Macneil et al. 2012; Meadows et al. 2012). Examples of theoretical frameworks include Bowlby's attachment theory and Erikson's life cycle stages (Kirby and Grover 2017). A formulation should be devised to assist with further assessment as well as with treatment planning; it may be particularly helpful for people who have not had an adequate response to traditional interventions and/or those with complex presentations and comorbidities (Macneil et al. 2012; Meadows et al. 2012). Formulation can be especially useful in identifying treatment challenges, such as difficulties in establishing a therapeutic alliance or ongoing problems of poor adherence to recommended treatments, and how to overcome these. Formulations are a living document which should be revisited to enable new information to be incorporated as it emerges (Macneil et al. 2012). 


\section{The Psychiatric Formulation in Mental Health Practice}

There are varied approaches to formulation. All attempt to address the question: Why has this patient presented in this manner at this time and why the condition (s) has/have continued (de Beer 2017). Although formulations can be biomedical in orientation, a common approach is to use the biopsychosocial model to frame the information gathering and synthesis. The "five Ps" constitute one popular approach to organizing the content of a formulation and are summarized by Macneil et al. (2012). Descriptions of variants of this approach are readily accessible elsewhere (e.g., Kirby and Grover 2017; Meadows et al. 2012). Thus, a two-dimensional grid may be generated to assist in synthesizing material using the five Ps along one axis and the biopsychosocial model along the other. In brief, the five Ps (often also referred to as the four P model if the initial summary is not included as one of the Ps) start with a summary of the presenting problem containing demographic details, relevant history and symptoms or behaviors, and how the person's life is affected. Next, predisposing factors such as biological contributors (such as genetic vulnerability which may be indicated by a family history of mental illness, acquired brain injury), psychological elements (such as the person's habitual coping style), and social factors (such as early childhood adversity, experience of migration as a child) are synthesized. Precipitating factors are then organized using the same biopsychosocial approach, and likely significant events preceding the onset of disorder are identified, such as stressors or losses. After this, perpetuating factors are summarized and encompass factors which may be hypothesized to maintain current problems or difficulties such as ongoing substance use, social isolation, chronic physical ill-health, prolonged unemployment, and poor engagement with services. The fifth $\mathrm{P}$ considers protective factors or the person's strengths which could include social supports, their motivation to change, their role and identity as a parent or employee, aspects of resilience, or helpful coping strategies.

Whatever the structure or approach used to generate a psychiatric formulation, all involve clinical reasoning skills which are underpinned by critical thinking and synthesis (de Beer 2017; Macneil et al. 2012; Meadows et al. 2012). As pointed out by de Beer (2017), step-by-step guidelines are lacking because psychiatric formulation is highly case specific; therefore, he has proposed that Bloom's taxonomy (an educational classification system) might be used to help teach formulation skills. Recognizing a similar lack of guidelines for psychosocial case formulation, Restifo (2010) proposed an initial set of psychosocial factors drawn from a range of theoretical perspectives which are accompanied by treatment implications. These include mismatch between coping skills and level of adversity; problematic beliefs, expectations, or wishes; disturbance of interpersonal attachments; "undigested" psychological material; and a systemic issue in which the disturbance is located with a group (see Restifo 2010 for further examples and details). Although still not commonplace, there are increasing calls for collaborative formulations which are co-constructed with consumers (Pemberton and Wainwright 2014). Similar care should be applied to developing collaborative formulations as to sharing diagnoses, due to the varied nature of consumers' reactions. 


\section{The Place of Culture within Diagnosis, Formulation, and the Biopsychosocial Model}

In acknowledgment of one of the shortcomings of diagnostic systems and standard approaches to formulation, there has been growing acceptance of the need for eliciting and organizing cultural information relevant to diagnostic assessment and treatment planning (see chapter $>$ "Psychological Perspective" for detailed consideration of social/cultural perspectives). This recognizes that culture affects the clinical encounter for every consumer, not only minority groups, since culture impacts psychiatric care through diverse influences on identity such as gender, age, and sexual orientation (Lewis-Fernandez et al. 2014). The biopsychosocial model is also sometimes elaborated to the biopsychosocial-cultural model to prompt consideration of cultural factors.

Mezzich et al. (2001) point out that cultural factors play a significant role at each stage of the data gathering process in psychiatric assessment. They highlight that activities, ranging from, for example, engaging appropriately with relatives and significant others, the manner of exploring intimate personal matters, the interpretation of patient reports concerning the significance and severity of emotional complaints, the evaluation of difficulties with social functioning, through to assessing the extent and value of social supports, are all impacted by culture. The Outline for Cultural Formulation (OCF) was introduced with DSM-4 to provide a framework for clinicians to organize cultural information relevant to diagnosis and treatment. The OCF organized clinical information in four domains: (1) cultural identity of the individual consumer; (2) cultural explanations of illness; (3) cultural interpretations of psychosocial stressors, supports, and levels of functioning; and (4) cultural aspects of the consumer-clinician relationship. DSM-5 introduced a Cultural Formulation Interview that operationalizes the data gathering process for the OCF and consists of a core 16-item questionnaire, reportedly taking about 20-25 min to complete (Lewis-Fernandez et al. 2014; Lewis-Fernandez et al. 2017).

\section{Beyond the Brain-Disease Model: Other Aetiological Theories of Mental IIIness}

\section{Socio-developmental Influences on the Genesis and Course of Mental Disorder: Accumulating Evidence}

In keeping with biopsychosocial approaches to causation and outcome of mental disorder, it is increasingly acknowledged that the disease risk associated with adverse environmental stimuli exceeds the effects of common genetic risk variants in exposed individuals by far (Meyer-Lindenberg and Tost 2012; Van Os et al. 2008). A plausible biopsychosocial model for the aetiology of depression is reasonably well accepted (Craig 2010). This builds on seminal work by the medical sociologist Professor George Brown and his colleagues who studied life events and difficulties which preceded the onset of depression in women (Brown and Harris 1978). 
As summarized by Craig (2010), up to $90 \%$ of depressive episodes are preceded by a severe life event within the previous months involving loss, humiliation (defined as likelihood that the event would provoke a sense of being put down or loss of selfesteem), and entrapment (defined as the extent to which the event underscored the fact of being trapped in a punishing situation). Subsequent studies have also identified psychosocial vulnerabilities such as negative self-evaluations and negative interactions in the home, which in the presence of a severe life event, were associated with depression onset (Craig 2010).

In relative contrast, evidence is still emerging to suggest that "adverse social contexts and experiences over the life course" are relevant to the onset of psychosis (Morgan and Hutchinson 2010). Studies examining whether social factors play a role in the onset of psychosis are limited by methodological challenges and the complexity of the enquiry. Nevertheless, strong and consistent evidence exists for elevated rates of schizophrenia and other psychoses in migrant and minority ethnic populations (Cantor-Graae 2007; Van Os et al. 2008). The overall relative risk for developing schizophrenia associated with migration is more than double (MeyerLindenberg and Tost 2012; van der Ven and Selten 2018). Cantor-Graae (2007) points out that this risk is greater than most other risk factors associated with schizophrenia, except for family history of psychosis; she concludes that these findings are hard to explain solely in terms of biological factors. Instead, they point to a role for social factors, although underlying mechanisms remain unclear (Cantor-Graae 2007; Morgan and Hutchinson 2010). A review of recent studies has proposed that migration or ethnic minority status are proxies for exposure to an inferior social status or discrimination, which remains the most credible explanation (van der Ven and Selten 2018). There is also reasonably consistent evidence for the association of urban environments with an approximately two- to threefold increased risk of schizophrenia (e.g., Cantor-Graae 2007; Meyer-Lindenberg and Tost 2012; Morgan and Hutchinson 2010; Van Os et al. 2008), although whether this is explained by social or other risk factors is yet to be established. At present, urbanicity represents an indicator of risk factors which are more prevalent in many densely populated environments (Cantor-Graae 2007; Morgan and Hutchinson 2010). Meyer-Lindenberg and Tost (2012) suggest that the more an individual stands out from their social milieu in terms of minority status, social fragmentation, and socioeconomic status, the higher their risk of developing schizophrenia. There is also emerging evidence to implicate childhood adversity, especially sexual, physical, and emotional abuse, in the onset of psychosis, although more longitudinal and population-based studies are needed (Bendall et al. 2008; Cantor-Graae 2007; Morgan and Hutchinson 2010). Further, early childhood adversity is an increasingly accepted risk factor for other severe mental illnesses (e.g., borderline personality disorder and depression) (Meyer-Lindenberg and Tost 2012).

Psychosocial influences on the course and outcome of disorders such as schizophrenia are well researched and recognized. For example, research has demonstrated a link between the emotional climate in families and the likelihood of relapse of a family member who has been diagnosed with schizophrenia (Farhall et al. 2012; Harvey and O'Hanlon 2013). Higher rates of relapse were observed in families 
where there was a high level of critical, hostile, or over-involved responses by family members toward the person living with schizophrenia (high expressed emotion), than in families where there was a low level of expressed emotion (Brown et al. 1972; Vaughn and Leff 1976). Such responses are best viewed as an understandable and common reaction to the experience of living with someone with a severe and relapsing condition (Harvey 2018). Arising from this research, family psychoeducational interventions were developed to reduce relapse by reducing high expressed emotion and helping family members to cope more effectively with the illness through education, stress reduction techniques, and skills training (Farhall et al. 2012; Harvey and O’Hanlon 2013).

\section{Current Understandings of Causation of Psychiatric Disorders: Gene- Environment Interactions and Epigenetics}

There is general agreement that there has been a failure to identify biomarkers for psychiatric diagnoses despite considerable research efforts, including studies of neuroimaging, cognitive neuroscience, and blood markers for cortisol suppression (e.g., Lacasse 2014b; Nemeroff et al. 2013; O'Donovan and Owen 2016; Timimi 2014). However, recent advances in genetic research have led to studies seeking to identify specific genetic markers for psychiatric disorders. The current state of knowledge concerning the genetics of psychiatric disorders is a vast and actively debated topic which can only be briefly summarized here.

The most significant advances have been made for schizophrenia with 50 genes identified compared with only 3 genes for anxiety disorder, according to a recent review of meta-analysis studies (Gatt et al. 2015). The emerging consensus is that most psychiatric disorders, including schizophrenia, bipolar disorder, and major depressive disorder, are complex genetically and highly polygenic (O'Donovan and Owen 2016). That is, they show evidence of the combined effects of many common genetic variants of small effect, as well as rare and de novo variants of large effect (Cattaneo et al. 2016; Gratten et al. 2014; O'Donovan and Owen 2016). Gatt et al. (2015) describe some examples of genetic variants specific to a disorder, but, overall, risk alleles tend not to be specific to any disorder (O'Donovan and Owen 2016). Thus, many of the individual genetic associations are shared across multiple disorders which points to extensive biological pleiotropy, that is, the altered function of a gene influences multiple traits (Gatt et al. 2015; O'Donovan and Owen 2016). An example of the cross-disorder effects of genetic variation comes from studies of copy number variants (CNVs) in which sections of the genome are repeated. These show that CNVs that influence risk for schizophrenia also often do so for autism spectrum disorders, intellectual disability, developmental delay, and attention deficit hyperactivity disorder (O’Donovan and Owen 2016). This is a rapidly evolving field in which current methodological limitations are likely to be incrementally addressed (Gatt et al. 2015).

It is now largely accepted that the best way to understand and integrate available evidence is to regard psychiatric disorders as being characterized by a complex 
interplay between genetic and environmental factors (Cattaneo et al. 2016; Van Os et al. 2008). Put another way, nature interacts with nurture to produce psychosis (and other psychiatric disorders) and not in a linear fashion but "where the effect of one is conditional on the other" (Van Os et al. 2008, p. 1066). Intriguingly, this echoes the systems thinking and reciprocal interactions described by Engel (1977) in the biopsychosocial model (Garland and Howard 2009). These developments should prompt social workers to rethink their knowledge base. In their influential paper, van Os et al. (2008) described that "biological synergism (co-participation of causes to some outcome) between environmental exposure and background genetic vulnerability is thought to be common in multifactorial disorders such as psychosis." Added to this, the rapidly growing science of epigenetics has become increasingly relevant. Epigenetics is the study of changes in organisms caused by modification of gene expression (active versus inactive genes) rather than alteration of the genetic code itself. That is, it concerns biological mechanisms that produce variability in phenotype (observed characteristics of an individual) without a change in genotype (their genetic sequence) (http://epialliance.org.au/what-is-epi/; Cattaneo et al. 2016; Garland and Howard 2009). In their recent overview of current knowledge, El-Sayed et al. (2013) summarize the main epigenetic mechanisms, which include DNA methylation, and report that epigenetic modification has been demonstrated in the aetiology of autism, schizophrenia, bipolar disorder, depression, anxiety disorders, and suicide. This extends our understanding of how both the environment and individual lifestyle can directly interact with an individual's genetic code to influence epigenetic change (http://epialliance.org.au/what-is-epi/), providing additional points of intervention on the pathway to development of psychiatric disorders.

\section{Conclusion}

The psychiatric contribution to mental health includes an emphasis on the importance of robust approaches to assessment and diagnosis which inform and underpin treatment and service delivery. Despite the limitations of the major diagnostic systems, diagnosis retains its central place in communicating a shared understanding of disordered experiences and behaviors between consumers, families, and professionals. Talking about a diagnosis in mental health is a skilled professional task, requiring sensitivity, an individualized approach within a trusting relationship, and the time to address and revisit concerns and misunderstandings. Helpful contributions can be made by all mental health professionals, including social workers.

The biopsychosocial (-cultural) model and psychiatric formulation, although not unique to psychiatry, are both noteworthy contributions to mental health practice from a medical perspective. Both help to complement and counterbalance the medical or brain-disease model of mental disorder which has been dominant in recent decades, and, consistent with social work theory and practice, they contribute strongly to a more person-centered, recovery-oriented approach to mental health practice which recognizes social aspects of human experience. Rapidly evolving understandings of aetiology in mental health, especially 
concerning gene-environment interactions and epigenetics, are consistent with these approaches. They help to integrate accumulating research evidence about social and environmental contributions to the development and course of mental disorders into aetiological theory and reaffirm the value of psychosocial interventions and personin-environment approaches to recovery. Some of the key implications for social work practice are summarized in Table 1.

\section{Cross-References}

Alcohol and Other Drug Use

- Community and Mental Health

- Ethics, Values and Recovery in Mental Health Social Work Practice

- Families and Mental Health

- General Introduction to Theories on Mental Health, Illness and Intervention

- Mental Health and Social Work - The Islamic Perspective

$>$ Overview and Reflection

$\checkmark$ Psychological Perspective

- Socio-Cultural and Ecological Perspective

- The Individual, Mental Health and Social Work

- Trauma-Informed Social Work Practice with Children and Youth

\section{References}

Altiere M, Von Kluge S (2009) Searching for acceptance: challenges encountered while raising a child with autism. J Intellect Develop Disabil 34(2):142-152

American Psychiatric Association (2013) Diagnostic and statistical manual of mental disorders, 5th edn. American Psychiatric Publishing, Arlington

Aminzadeh F, Byszewski A, Molnar F, Eisner M (2007) Emotional impact of dementia diagnosis: exploring persons with dementia and caregivers' perspectives. Aging Ment Health 11(3):281-290

Bendall S, Jackson H, Hulbert C, McGorry P (2008) Childhood trauma and psychotic disorders: a systematic, critical review of the evidence. Schizophr Bull 34(3):568-579

Bland R et al (2012) Assessment: specialised assessment skills. In: Meadows G, Grigg M, Farhall J, McDermott F, Fossey E, Singh B (eds) Mental health in Australia: collaborative community practice, 3rd edn. Oxford University Press, Sydney, pp 394-400

Borrell-Carrió F, Suchman A, Epstein R (2004) The biopsychosocial model 25 years later: principles, practice, and scientific inquiry. Ann Fam Med 2(6):576-582

Brown G, Harris T (1978) Social origins of depression: a study of psychiatric disorder in women. Tavistock Publications Ltd, London

Brown G, Birley J, Wing J (1972) Influence of family life on the course of schizophrenic disorders: a replication. Br J Psychiatry 121:241-258

Buston K (2002) Adolescents with mental health problems: what do they say about health services? J Adolesc 25:231-242

Cantor-Graae E (2007) The contribution of social factors to the development of schizophrenia: a review of recent findings. Can J Psychiatr 52:277-286 
Cattaneo A, Cattane N, Begni V, Pariante C, Riva M (2016) The human BDNF gene: peripheral gene expression and protein levels as biomarkers for psychiatric disorders. Transl Psychiatry 6 (11):e958

Chaplin R, Lelliott P, Quirk A, Seale C (2007) Negotiating styles adopted by consultant psychiatrists when prescribing antipsychotics. Adv Psychiatr Treat 13:43-50

Craig T (2010) Depression. In: Morgan C, Bhugra D (eds) Principles of social psychiatry. Wiley, Chichester, pp 215-226

de Beer W (2017) Original opinion: the use of bloom's taxonomy to teach and assess the skill of the psychiatric formulation during vocational training. Australas Psychiatry 25(5):514-519

Davis K, Kahn R, Ko G et al (1991) Dopamine in schizophrenia: A review and reconceptualization. American Journal of Psychiatry 148:1474-1486

Dinos S, Stevens S, Serfaty M, Weich S, King M (2004) Stigma: the feelings and experiences of 46 people with mental illness. Qualitative study. Br J Psychiatry 184:176-181

Elam M (2015) How the brain disease paradigm remoralizes addictive behaviour. Sci Cult 24(1):46-64

El-Sayed A, Koenen K, Galea S (2013) Putting the "epi” into epigenetics research in psychiatry. J Epidemiol Community Health 67(7):610-616

Engel G (1977) The need for a new medical model: a challenge for biomedicine. Science 196:129-136

Farhall J et al (2012) Psychological therapies. In: Meadows G, Farhall J, Fossey E, Grigg M, McDermott F, Singh B (eds) Mental health in Australia. Oxford University Press, South Melbourne, pp 468-501

Farre A, Rapley T (2017) The new old (and old new) medical model: four decades navigating the biomedical and psychosocial understandings of health and illness. Healthcare 5(4):88

Fava G, Sonino N (2008) The biopsychosocial model thirty years later. Psychother Psychosom 77(1):1-2

Ferentzy P, Turner N (2012) Morals, medicine, metaphors, and the history of the disease model of problem gambling. J Gambl Issues 27:1-27

Fossey E, Harvey C, Mokhtari M, Meadows G (2012) Self-rated assessment of needs for mental health care: a qualitative analysis. Community Ment Health J 48(4):407-419

Frances A (2011) Psychosis risk syndrome - far too risky. Aust N Z J Psychiatry 45(10):803-804

Frances A (2012) DSM 5 Is a guide not a bible - ignore its ten worst changes: APA approval of DSM-5 is a sad day for psychiatry. Psychology Today, US

Gallagher A, Arber A, Chaplin R, Quirk A (2010) Service users' experience of receiving bad news about their mental health. J Ment Health 19(1):34-42

Garland E, Howard M (2009) Neuroplasticity, psychosocial genomics, and the biopsychosocial paradigm in the 21st century. Health Soc Work 34(3):191-199

Gatt J, Burton K, Williams L, Schofield P (2015) Specific and common genes implicated across major mental disorders: a review of meta-analysis studies. J Psychiatr Res 60:1-13

Ghaemi S (2009) The rise and fall of the biopsychosocial model. Br J Psychiatry 195:3-4

Gratten J, Wray N, Keller M, Visscher P (2014) Large-scale genomics unveils the genetic architecture of psychiatric disorders. Nat Neurosci 17(6):782-790

Harvey C (2018) Family psychoeducation for people living with schizophrenia and their families. Br J Psychiatr Adv 24(1):9-19

Harvey C, O'Hanlon B (2013) Family psycho-education for people with schizophrenia and other psychotic disorders and their families. Aust N Z J Psychiatry 47:516-520

Healy K (2016) After the biomedical technology revolution: where to now for a bio-psycho-social approach to social work? Br J Soc Work 46(5):1446-1462

Holm-Denoma J et al (2008) Patients' affective reactions to receiving diagnostic feedback. J Soc Clin Psychol 27:555-575

Howes O, McCutcheon R, Owen M, Murray R (2017) The role of genes, stress and dopamine in the development of schizophrenia. Biol Psychiatry 81(1):9-20 
Humphreys C, Thiara R (2003) Mental health and domestic violence: "I call it symptoms of abuse". Br J Soc Work 33:209-226

Kendell R, Jablensky A (2003) Distinguishing between the validity and utility of psychiatric diagnoses. Am J Psychiatry 160(1):4-12

Kendler K, Schaffner K (2011) The dopamine hypothesis of schizophrenia: an historical and philosophical analysis. Philos Psychiatry Psychol 18(1):41-63

Kirby K, Grover A (2017) The psychiatric interview, mental state examination and formulation. In: Bloch S, Green S, Janca A, Mitchell P, Robertson M (eds) Foundations of clinical psychiatry, 4th edn. Melbourne University Press, Melbourne

Lacasse J (2014a) A critical appraisal of the DSM-5: social work perspectives [Special Issue]. Res Soc Work Pract 24(1):5-173

Lacasse J (2014b) After DSM-5: a critical mental health research agenda for the 21st century. Res Soc Work Pract 24(1):5-10

Lau C, Wang H, Hsu J, Liu M (2013) Does the dopamine hypothesis explain schizophrenia? Rev Neurosci 24(4):389-400

Leff J, Sartorius N, Jablensky A, Korten A, Ernberg G (1992) The international pilot study of schizophrenia: five-year follow-up findings. Psychol Med 22:131-145

Lewis-Fernandez R et al (2014) Culture and psychiatric evaluation: operationalizing cultural formulation for DSM-5. Psychiatry 77(2):130-154

Lewis-Fernandez R et al (2017) Feasibility, acceptability and clinical utility of the cultural formulation interview: mixed-methods results from the DSM-5 international field trial. Br J Psychiatry 210:290-297

Lundahl B, Kunz C, Brownell C, Tollefson D, Burke B (2010) A meta-analysis of motivational interviewing: twenty-five years of empirical studies. Res Soc Work Pract 20:137-160

Macneil C, Hasty M, Conus P, Berk M (2012) Is diagnosis enough to guide interventions in mental health? Using case formulation in clinical practice. BMC Med 10:111

Magliano L, Fiorillo A, Malangone C, Vecchio H, Maj M (2008) Views of persons with schizophrenia on their own disorder: an Italian participatory study. Psychiatr Serv 59(7):795-799

Malla A, Joober R, Garcia A (2015) Mental illness is like any other medical illness: a critical examination of the statement and its impact on patient care and society. J Psychiatry Neurosci 40(3):147-150

Maynard B, Boutwell B, Vaughn M (2017) Advancing the science of social work: the case for biosocial research. Br J Soc Work 47(5):108

Meadows G et al (2012) Assessment: essential skills. In: Meadows G, Grigg M, Farhall J, McDermott F, Fossey E, Singh B (eds) Mental health in Australia: collaborative community practice, 3rd edn. Oxford University Press, Sydney, pp 342-393

Meltzer H, Stahl S (1976) The dopamine hypothesis of schizophrenia: a review. Schizophr Bull 2(1):19-76

Mestdagh A, Hansen B (2014) Stigma in patients with schizophrenia receiving community mental health care: a review of qualitative studies. Soc Psychiatry Psychiatr Epidemiol 49(1):79-87

Meyer-Lindenberg A, Tost H (2012) Neural mechanisms of social risk for psychiatric disorders. Nat Neurosci 15(5):663-668

Mezzich J, Berganza C, Ruiperez M (2001) Culture in DSM-IV, ICD-10, and evolving diagnostic systems. Psychiatr Clin N Am 24(3):407-419

Milton A, Mullan B (2014) Communication of a mental health diagnosis: a systematic synthesis and narrative review. J Ment Health 23(5):261-270

Milton A, Mullan B (2017) Views and experience of communication when receiving a serious mental health diagnosis: satisfaction levels, communication preferences, and acceptability of the SPIKES protocol. J Ment Health 26(5):395-404

Morgan C, Bhugra D (2010) Principles of social psychiatry. Wiley, Chichester

Morgan C, Hutchinson G (2010) The sociodevelopmental origins of psychosis. In: Morgan C, Bhugra D (eds) Principles of social psychiatry. Wiley, Chichester, pp 193-213

Morse S (2004) Medicine and morals, craving and compulsion. Subst Use Misuse 39(3):437-460 
National Institute for Health and Care Excellence (2009) NICE guideline. Borderline personality disorder: recognition and management. National Institute for Health and Care Excellence, British Psychological Society, UK.

Nemeroff C et al (2013) DSM-5: a collection of psychiatrist views on the changes, controversies, and future directions. BMC Med 11:202

Nesse R, Stein D (2012) Towards a genuinely medical model for psychiatric nosology. BMC Med 10:5

O'Donovan M, Owen M (2016) The implications of the shared genetics of psychiatric disorders. Nat Med 22(11):1214-1219

Outram S et al (2014) Communicating a schizophrenia diagnosis to patients and families: a qualitative study of mental health clinicians. Psychiatr Serv 65(4):551-554

Peele S (2016) People control their addictions. No matter how much the chronic brain disease model of addiction indicates otherwise, we know that people can quit addictions - with special reference to harm reduction and mindfulness. Addict Behav Rep 4:97-101

Pemberton R, Wainwright T (2014) The end of mental illness thinking? Int J Clin Health Psychol 14(3):216-220

Regier D, Narrow W, Clarke D, Kraemer H, Kuramoto S, Kuhl E, Kupfer D (2013) DSM-5 field trials in the United States and Canada, Part II: test-retest reliability of selected categorical diagnoses. Am J Psychiatry 170(1):59-70

Restifo S (2010) An empirical categorization of psychosocial factors for clinical case formulation and treatment planning. Australas Psychiatry 18(3):210-213

Rutter M (2011) Research review: child psychiatric diagnosis and classification: concepts, findings, challenges and potential. J Child Psychol Psychiatry 52:647-660

Singh B, Ng C, Meadows G (2012) The global perspective. In: Meadows G, Grigg M, Farhall J, McDermott F, Fossey E, Singh B (eds) Mental health in Australia: collaborative community practic, 3rd edn. Oxford University Press, Sydney, pp 31-37

Smith R, Fortin A, Dwamena F, Frankel R (2013) An evidence-based patient-centered method makes the biopsychosocial model scientific. Patient Educ Couns 91(3):265-270

Stafford M, Jackson H, Mayo-Wilson E et al (2013) Early interventions to prevent psychosis: Systematic review and meta-analysis. BMJ 346:f185

Teesson M, Slade T, Mills K (2009) Comorbidity in Australia: findings of the 2007 National Survey of Mental Health and Wellbeing. Aust N Z J Psychiatry 43:606-614

Timimi S (2014) No more psychiatric labels: why formal psychiatric diagnostic systems should be abolished. Int J Clin Health Psychol 14(3):208-215

Timimi S, Thomas P, Davies J, Kinderman P (2014) Antipsychiatry and the antidepressants debate Lancet. Psychiatry 1(3): 174

Tyrer P, Crawford M, Mulder R (2011) Reclassifying personality disorders. Lancet 377(9780):1814-1815

van der Ven E, Selten J (2018) Migrant and ethnic minority status as risk indicators for schizophrenia: new findings. Curr Opin Psychiatry 31(3):231-236

van Os J, Guloksuz S (2017) A critique of the "ultra-high risk" and "transition" paradigm. World Psychiatry 16(2):200-206

van Os J, Murray RM (2013) Can we identify and treat "schizophrenia light" to prevent true psychotic illness? Br Med J 346:f304

Van Os J, Rutten B, Poulton R (2008) Gene-environment interactions in schizophrenia: review of epidemiological findings and future directions. Schizophr Bull 34(6):1066-1082

Vaughn C, Leff J (1976) The influence of family and social factors on the course of psychiatric illness. A comparison of schizophrenic and depressed neurotic patients. Br J Psychiatry 129:125-137

Wakefield J (2013) DSM-5 and clinical social work: mental disorder and psychological justice as goals of clinical intervention. Clin Soc Work J 41(2):131-138 
Wong S (2014) A critique of the diagnostic construct schizophrenia. Res Soc Work Pract 24(1):132-141

World Health Organisation (1992) The ICD-10 classification of mental and behavioural disorders. World Health Organisation, Geneva

World Health Organisation (2001) International Classification of Functioning, disability and health (ICF). World Health Organisation, Geneva

Yung A, Nelson B (2013) The ultra-high risk concept-a review. Can J Psychiatr 58(1):5-12 